\title{
Perioperative outcomes of robotic pancreaticoduodenectomy: a single surgeon's experience with 55 consecutive cases
}

\author{
Ronggui Lin, Xianchao Lin, Maoen Pan, Fengchun Lu, Yuanyuan Yang, Congfei Wang, Haizong Fang, \\ Yanchang Chen, Heguang Huang \\ Department of General Surgery, Fujian Medical University Union Hospital, Fuzhou, China \\ Contributions: (I) Conception and design: R Lin, H Huang; (II) Administrative support: F Lu, Y Chen; (III) Provision of study materials or patients: \\ All authors; (IV) Collection and assembly of data: X Lin, M Pan, Y Yang; (V) Data analysis and interpretation: C Wang, H Fang; (VI) Manuscript \\ writing: R Lin; (VII) Final approval of manuscript: All authors. \\ Correspondence to: Heguang Huang. Department of General Surgery, Fujian Medical University Union Hospital, 29 Xinquan Road, Fuzhou 350001, \\ China. Email: Heguanghuang2@163.com.
}

\begin{abstract}
Backgrounda Robotic pancreaticoduodenectomy (RPD) has been increasingly performed for patients with periampullary tumours and tumours in the pancreatic head. This method offers several technical advantages compared to open and laparoscopic surgeries. However, the surgical results often vary depending on the experience of different pancreatic centres.

Methods: A retrospective study of our first 55 cases of RPD from August 2016 to April 2020 was conducted to evaluate the perioperative outcomes of RPD and to summarize the operative experiences in a single intuition. Benign and malignant tumours in the pancreatic head or periampullary tumours without obvious vascular and adjacent organ invasion were included in this study. Perioperative characteristics and postoperative complications of the enrolled patients were retrospectively collected.

Results: The first 17 cases were robot-assisted laparoscopic pancreaticoduodenectomy (RA-LPD) and the remaining 38 patients underwent total RPD. The RA-LPD group had a remarkably longer operative time than the total RPD group $(415.3 \pm 89.2$ vs. $362.4 \pm 75.6 \mathrm{~min}, \mathrm{P}=0.047)$. The incidences of biliary leakage, chyle leakage, DGE, intra-abdominal infection and intra-abdominal haemorrhage were 3.6\%, 0.0\%, 5.5\%, 9.1\% and $5.5 \%$, respectively. Two patients underwent relaparotomy due to severe intra-abdominal haemorrhage. The median length of hospital stay was 14 (11 to 19) days. There were no deaths during the perioperative period.
\end{abstract}

Conclusions: RPD is a technically feasible procedure for selected patients with periampullary tumours and tumours in the pancreatic head in experienced hands.

Keywords: Robotic; pancreaticoduodenectomy; outcome

Submitted Jun 10, 2020. Accepted for publication Nov 06, 2020.

doi: $10.21037 / g s-20-552$

View this article at: http://dx.doi.org/10.21037/gs-20-552

\section{Introduction}

Pancreaticoduodenectomy (PD) is a classic procedure for benign and malignant periampullary tumours and tumours in the pancreatic head. In 1994, Gagner et al. (1) reported the first laparoscopic pancreaticoduodenectomy (LPD) in the world. Giulianotti et al. (2) was the first in the world to perform robotic pancreaticoduodenectomy (RPD) in 2001 and reported it in 2003. Since then, minimally invasive pancreaticoduodenectomy (MIPD), including LPD and $\mathrm{RPD}$, has been increasingly performed worldwide in recent decades $(3,4)$. Nassour et al. (5) demonstrated that MIPD had comparable morbidity and mortality rates compared to open pancreaticoduodenectomy (OPD), indicating the safety of this technique and its satisfactory surgical outcomes. Yin et al. (6) reported that the surgical and oncological outcomes showed no differences between LPD 
and OPD for pancreatic ductal adenocarcinoma (PDAC). A recent meta-analysis by Podda et al. (7) demonstrated that oncological outcomes, including the number of harvested lymph nodes and positive margin rate, were equivalent between RPD and OPD, and RPD had a longer operative time and less estimated blood loss.

However, LPD has some technical shortages, including restricted caudal-to-cranial vision and a limited range of surgical instrument motion, difficulties in hepaticojejunostomy and pancreaticojejunostomy, especially when encountering a small bile duct or pancreatic duct, and has a steep learning curve. Compared to the laparoscope, the robotic system provides a 10- to 15 -fold more magnified three-dimensional vision, seven degrees of freedom, and precise movement of the surgical instruments and eliminates hand tremors. The advantages are beneficial for gentle and precise manipulation for dissection and suturing, providing easier separation of the tissue and major vasculature and better control of unexpected bleeding than laparoscopy; thus, this method is especially suitable for technically demanding pancreaticoduodenectomy (8).

The robotic operative platform has attracted increasing interest from pancreatic surgeons because it overcomes many shortages of the laparoscopic system, and RPD has a shorter learning curve than LPD. However, RPD has not yet been widely performed worldwide. The increased medical cost may be the main reason for the limited use of RPD. The complexity of the procedure and lack of standardized protocol also hinder its application in clinical practice. Moreover, different characteristics and operative processes between the robotic and laparoscopic systems slow down the transition from LPD to RPD in some centres. However, only a few high-volume pancreatic centres have reported the operative technique of RPD in detail as well as their initial experience.

A consecutive series of patients with benign and malignant periampullary tumours and tumours in the pancreatic head who underwent RPD in our department were enrolled in the present study. This study aimed to evaluate the surgical and oncological outcomes of our standard operative process and modified technique for pancreaticojejunostomy. We present the following article in accordance with the STROBE reporting checklist (available at http://dx.doi.org/10.21037/gs-20-552).

\section{Methods}

A retrospective study of patients who underwent RPD between August 2016 and April 2020 was conducted. All operations were carried out by a group of surgeons with extensive experience in laparoscopic and open pancreatic surgeries in a high-volume pancreatic centre in China. This group of surgeons had performed over 50 cases of LPD before the first case of RPD. The study was conducted in accordance with the Declaration of Helsinki (as revised in 2013). The study protocol was approved by the ethics committee of Fujian Medical University Union Hospital (2020KY0126). Written informed consent was obtained from the patients for publication of this manuscript and any accompanying images.

The inclusion criteria were benign and malignant tumours in the pancreatic head or periampullary tumours without obvious vascular and adjacent organ invasion. The exclusion criteria were as follows: locally advanced tumours with vascular invasion or those requiring adjacent organ resection, and distant metastasis.

\section{Clinicopathological characteristics of the patients}

Preoperative, perioperative and postoperative characteristics of the patients enrolled in this study were retrospectively collected. The preoperative and perioperative characteristics of the patients mainly included age, sex, body mass index (BMI), comorbidities, American Society of Anesthesiologists (ASA) score, serum levels of albumin (ALB), alanine aminotransferase (ALT), aspartate aminotransferase (AST), and total bilirubin (TBIL), obstructive jaundice, preoperative percutaneous transhepatic cholangial drainage (PTCD), tumour location, pathological type, largest tumour diameter, number of lymph nodes harvested, operation time, estimated blood loss (EBL) and blood transfusion. The postoperative characteristics included postoperative complications and length of hospital stay (LOS). Clinically relevant postoperative pancreatic fistula (CR-POPF), chyle leakage, delayed gastric emptying (DGE), and intraabdominal haemorrhage were defined by the International Study Group on Pancreatic Surgery (ISGPS) (9-12).

\section{Patient position, trocar placement and docking}

The robotic operations were performed with the Da Vinci Si Surgical System (Intuitive Surgical, Sunnyvale, CA, USA). The patients were placed in a supine $20^{\circ}$ reverse Trendelenburg position with their legs apart and a slight left-side tilt. The assistant surgeon stood between the patient's legs. A $12-\mathrm{mm}$ camera port was placed below 


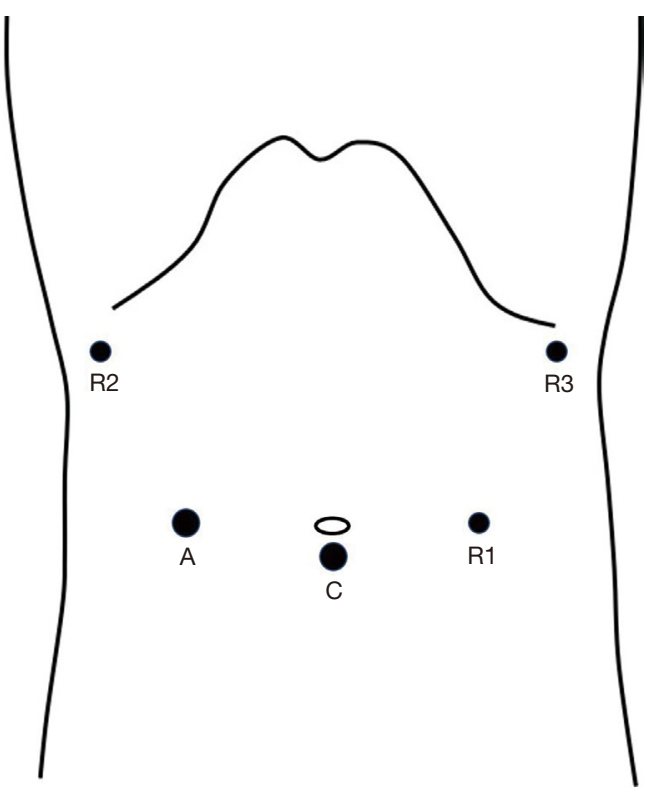

Figure 1 Trocar placements in robotic pancreaticoduodenectomy (RPD). A, assistant port; C, camara port; R1, the first robotic arm; $\mathrm{R} 2$, the second robotic arm; R3, the third robotic arm.

the umbilicus (Figure 1). Following the induction of pneumoperitoneum, the scope was inserted to explore the abdominal cavity to exclude distant metastasis before docking. The robotic system was then docked at the head side of the patient. An 8-mm trocar was placed at the crosspoint between the horizontal line of the umbilicus and the left mid-clavicular line for R1 (first robotic arm). R2 (second robotic arm) was placed at the right anterior axillary line $2-3 \mathrm{~cm}$ below the ribs. A $12-\mathrm{mm}$ assistant trocar and R3 (third robotic arm) were located at the opposite positions of R1 and R2 on the contralateral side, respectively. Interference between the adjacent robotic arms was prevented by keeping a distance of $10-15 \mathrm{~cm}$.

The gastrocolic ligament was opened for preliminary exploration of the pancreas. The hepatic colonic flexure, right transverse colon and mesocolon were mobilized downward to visualize the duodenum and the pancreatic head fully. The inferior edge of the pancreas was dissected to expose the superior mesenteric vein (SMV), and the retropancreatic space was then separated. Later, the gastrocolic trunk (GCT) was ligated. Part of the distal stomach was resected with $60 \mathrm{~mm}$ linear cutter staplers (Echelon, Johnson \& Johnson, USA). The superior edge of the pancreas was dissected to expose the common hepatic artery (CHA). The right gastric artery (RGA) and the gastroduodenal artery (GDA) were ligated at the root, and the portal vein (PV) was then exposed. The common hepatic duct was divided after resection of the gallbladder, followed by skeletonization of the hepatoduodenal ligament. A rightposterior "artery-first" approach was performed to expose the superior mesenteric artery (SMA). An extended Kocher manoeuvre was carried out to dissect and then retract the duodenum and the pancreatic head medially to gain visualization of the aorta (AA) and the left renal vein (LRC). The SMA was identified and dissected approximately $1 \mathrm{~cm}$ superiorly to the LRC. The posterior adhesion between the uncinate process of the pancreas and the SMA was separated, and the inferior pancreaticoduodenal artery (IPDA) was ligated. The pancreas was then divided at the neck cranially. The proximal jejunum was pulled to the right upper quadrant and divided with a $60 \mathrm{~mm}$ linear cutter stapler. The final step of resection was the separation of the uncinate process from the SMV and the SMA. The uncinate process became thinner after dissection via the right-posterior "SMA-first" approach. The pancreatic head and duodenum were retracted laterally with a 45$60^{\circ}$ anticlockwise rotation, and the SMV was retracted medially to provide better visualisation during dissection of the uncinate process. The uncinate process was then separated cranially and longitudinally along the right aspect of the SMA. The right $180^{\circ}$ of the SMA was cleared with en bloc resection of the specimen (as indicated in Video 1). A 5-cm curved periumbilicus incision was made to remove the specimen, and the robotic system was subsequently redocked.

Digestive reconstruction was carried out with the modified Child's technique. A modified double-layer pancreaticojejunostomy with the duct-to-mucosa technique was performed in our centre. The outside layer of the anastomosis was a continuous suture with 4-0 prolene between the seromuscular layer of the jejunum and was located $0.5 \mathrm{~cm}$ to the margin of the pancreatic remnant, which can partly enwrap the stump of the pancreas after anastomosis. The inside layer is a duct-to-mucosa anastomosis, with suturing from the margin of the pancreatic stump to the main pancreatic duct (MPD) and to the corresponding point of the jejunum. This method prevented tearing the pancreatic duct and parenchyma and eliminated the potential space between the jejunum and pancreatic remnant with better attachment. Six to eight stitches were often required to accomplish the ductto-mucosa anastomosis, and a trans-anastomotic stent 
Table 1 The clinicopathological characteristics of patients underwent RPD

\begin{tabular}{|c|c|}
\hline Variables & Patients $(n=55)$ \\
\hline Age (years) & $51.5 \pm 11.7$ \\
\hline Gender (male/female) & $25 / 30$ \\
\hline \multicolumn{2}{|l|}{ Comorbidity } \\
\hline Hypertension & 3 \\
\hline Diabetes & 5 \\
\hline BMI $\left(\mathrm{kg} / \mathrm{m}^{2}\right)$ & $22.2 \pm 2.4$ \\
\hline \multicolumn{2}{|l|}{ ASA score } \\
\hline 1 & 18 \\
\hline II & 34 \\
\hline III & 3 \\
\hline Serum albumin level (g/L) & $42.0 \pm 4.9$ \\
\hline Serum ALT level (U/L), median (IQR) & $31.0(17.0-100.0)$ \\
\hline Serum AST level (U/L), median (IQR) & $27.0(17.0-51.0)$ \\
\hline Serum TBIL level ( $\mu \mathrm{mol} / \mathrm{L})$, median (IQR) & $15.2(9.5-38.0)$ \\
\hline Obstructive jaundice & 16 \\
\hline Preoperative PTCD & 10 \\
\hline \multicolumn{2}{|l|}{ Tumor location } \\
\hline Pancreatic head & 32 \\
\hline Duodenum & 15 \\
\hline Ampulla & 8 \\
\hline \multicolumn{2}{|l|}{ Type of pathology } \\
\hline Benign & 31 \\
\hline Malignant & 24 \\
\hline Largest tumor diameter $(\mathrm{cm})$ & $3.0 \pm 1.5$ \\
\hline
\end{tabular}

RPD, robotic pancreaticoduodenectomy; BMI, body mass index; ASA, American Society of Anesthesiologists; TBIL, total bilirubin; ALT, alanine aminotransferase; AST, aspartate aminotransferase; IQR, inter-quartile; PTCD, percutaneous transhepatic cholangial drainage.

was routinely placed (as indicated in Video 2). An end-toside hepaticojejunostomy was performed with interrupted or continuous sutures depending on the diameter of the common hepatic duct. Gastrojejunostomy was performed between the posterior wall of the stomach and the jejunum with a side-to-side anastomosis technique using a $60 \mathrm{~mm}$ linear cutter stapler (Echelon, Johnson \& Johnson).

\section{Statistical analysis}

Continuous variables are presented as mean $\pm \mathrm{SD}$ or median with inter-quartile (IQR). Categorical variables are presented as numbers and percentages. Comparison of perioperative factors between two groups were conducted using the two independent samples t-test, Mann-Whitney $\mathrm{U}$ test, Chi-square test, or Fisher exact test. $\mathrm{P}$ value $<0.05$ was considered as statistical significance. All statistical analysis was performed with SPSS statistical software (version 22.0, IBM Corp).

\section{Results}

Fifty-five consecutive patients with periampullary tumours or tumours in the pancreatic head who underwent RPD from August 2016 to April 2020 were eventually enrolled in this study. The clinicopathological characteristics of the patients are shown in Table 1. There were 25 male patients and 30 female patients. The mean age and BMI were $51.5 \pm$ 11.7 years and $22.2 \pm 2.4 \mathrm{~kg} / \mathrm{m}^{2}$, respectively. The serum levels of ALT, AST, ALB and TBIL were 31.0 (IQR, 17.0100.0) U/L, 27.0 (IQR, 17.0-51.0) U/L, 15.2 (IQR, 9.538.0) $\mathrm{g} / \mathrm{L}$, and $42.0 \pm 4.9 \mu \mathrm{mol} / \mathrm{L}$, respectively. There were 32 patients with tumours in the pancreatic head, 15 patients with duodenal tumours, and 8 patients with ampullary tumours. Thirty-one tumours were benign, and 24 tumours were malignant, with the largest tumour diameter being $3.0 \pm 1.5 \mathrm{~cm}$. Sixteen patients manifested obstructive jaundice, and ten patients underwent preoperative biliary drainage.

The first 17 cases were robot-assisted LPD (RA-LPD) with laparoscopic resection and robotic reconstruction. The remaining 38 patients underwent total RPD with robotic resection and reconstruction. To evaluate the efficacy of RALPD $v s$. total RPD procedures during the resection period, the perioperative factors of both groups were compared (as shown in Table 2). The RA-LPD group had a remarkably longer operative time than the total RPD group $(415.3 \pm 89.2$ vs. $362.4 \pm 75.6 \mathrm{~min}, \mathrm{P}=0.047)$. The median $\mathrm{EBL}$ in the total RPD group [200 (IQR, 100-400) $\mathrm{mL}$ ] seemed to be less than that in the RA-LPD group [300 (IQR, 75-500) mL], although there was no significant difference $(\mathrm{P}=0.439)$. No significant differences existed in the number of harvested lymph nodes $(13.6 \pm 4.0 v$ vs. 14.2 \pm 5.7$)$ or blood transfusions (2 $v s$. 2) between the RA-LPD and total RPD groups $(\mathrm{P}>0.05)$.

Clinical risk factors and scores related to postoperative 
Table 2 Comparison of perioperative factors between RA-LPD and total RPD group

\begin{tabular}{lccc}
\hline Variables & $\begin{array}{c}\text { RA-LPD } \\
(\mathrm{n}=17)\end{array}$ & $\begin{array}{c}\text { Total RPD } \\
(\mathrm{n}=38)\end{array}$ & P value \\
\hline $\begin{array}{l}\text { Harvested Lymph } \\
\text { nodes }\end{array}$ & $13.6 \pm 4.0$ & $14.2 \pm 5.7$ & 0.698 \\
Operative time (min) & $415.3 \pm 89.2$ & $362.4 \pm 75.6$ & 0.047 \\
$\begin{array}{l}\text { EBL }(\mathrm{mL}), \text { median } \\
{[\mathrm{QQR}]}\end{array}$ & $300[75-500]$ & $200[100-400]$ & 0.439 \\
Blood transfusion & 2 & 2 & 0.579 \\
\hline
\end{tabular}

RPD, robotic pancreaticoduodenectomy; RA-LPD, robot-assisted laparoscopic pancreaticoduodenectomy; EBL, estimated blood loss; IQR, inter-quartile.

pancreatic fistula (13) are shown in Table 3. The clinical risk scores for postoperative pancreatic fistula were as follows: negligible risk ( 0 points, 3 cases), low risk (1-2 points, 6 cases), intermediate risk (3-6 points, 43 cases), and high risk (7-10 points, 3 cases).

The postoperative complications and factors after RPD are shown in Table 4. There was 1 case of conversion to separate the adhesion between the tumour and the PV/ $\mathrm{SMV}$. The overall incidence of CR-POPF (grade $\mathrm{B}+\mathrm{C}$ ) was $12.7 \%$, which includes $9.1 \%$ grade $\mathrm{B}$ and $3.6 \%$ grade $\mathrm{C}$ of CR-POPF. The incidences of biliary leakage, chyle leakage, DGE, intra-abdominal infection and intra-abdominal haemorrhage were $3.6 \%, 0.0 \%, 5.5 \%, 9.1 \%$ and $5.5 \%$, respectively. Two patients underwent relaparotomy due to severe intra-abdominal haemorrhage. The median length of hospital stay was 14 (IQR, 11-19) days. There were no deaths during the perioperative period.

\section{Discussion}

Several studies have reported that the morbidity and mortality of RPD were comparable to those of OPD and LPD. A propensity-matched study by Wang et al. with 87 cases of RPD showed that RPD led to less blood loss, more harvested lymph nodes, a lower incidence of DGE, and a similar rate of POPF (14) than OPD. A recent metaanalysis by Yan et al. showed that RPD had equivalent outcomes, including harvested lymph nodes, positive margin rate, postoperative complications and mortality rate, to OPD (8). Another recent meta-analysis by Kamarajah et al. analysing 1,025 RPD and 2,437 LPD procedures showed that RPD had an equivalent rate of postoperative complications and $\mathrm{R} 0$ resection rate but a lower conversion
Table 3 Clinical risk factors and scores related to postoperative pancreatic fistula after RPD

\begin{tabular}{|c|c|c|}
\hline Risk factor, parameter & Patients $(n=55)$ & Points \\
\hline \multicolumn{3}{|l|}{ Pancreatic texture } \\
\hline Firm/hard & 20 & 0 \\
\hline Soft & 35 & 2 \\
\hline \multicolumn{3}{|l|}{ MPD diameter (mm) } \\
\hline$\geq 5$ & 5 & 0 \\
\hline 4 & 12 & 1 \\
\hline 3 & 16 & 2 \\
\hline 2 & 18 & 3 \\
\hline$\leq 1$ & 4 & 4 \\
\hline \multicolumn{3}{|l|}{$\mathrm{EBL}(\mathrm{mL})$} \\
\hline$\leq 400$ & 47 & 0 \\
\hline $400-700$ & 4 & 1 \\
\hline $700-1,000$ & 1 & 2 \\
\hline$>1,000$ & 3 & 3 \\
\hline \multicolumn{3}{|l|}{ Pathology } \\
\hline PDAC or chronic pancreatitis & 13 & 0 \\
\hline All others & 42 & 1 \\
\hline \multicolumn{3}{|l|}{ CRS-POPF } \\
\hline Negligible risk & 3 & 0 \\
\hline Low risk & 6 & $1-2$ \\
\hline Intermediate risk & 43 & $3-6$ \\
\hline High risk & 3 & $7-10$ \\
\hline
\end{tabular}

RPD, robotic pancreaticoduodenectomy; MPD, main pancreatic duct; EBL, estimated blood loss; PDAC, pancreatic adenocarcinoma; CRS-POPF, clinical risk scores for postoperative pancreatic fistula.

rate and transfusion rate than LPD (15). Kowalsky et al. demonstrated that the combination of RPD and enhanced recovery after surgery (ERAS) significantly decreased the LOS and overall cost compared with OPD (16). The studies above strongly supported the safety and efficacy of RPD compared to OPD and LPD.

The indications for RPD for periampullary tumours and tumours in the pancreatic head have been extended in some centres, even for those with vascular involvement (17). Beane et al. conducted a retrospective review of 380 patients who underwent RPD, including 50 cases of combined 
Table 4 Postoperative complications and factors after RPD

\begin{tabular}{lc}
\hline Postoperative complications and factors & Patients $(\mathrm{n}=55)$ \\
\hline CR-POPF (Grade B+C) & $7(12.7 \%)$ \\
Grade B & $5(9.1 \%)$ \\
Grade C & $2(3.6 \%)$ \\
Biliary leakage & $2(3.6 \%)$ \\
Chyle leak & $0(0.0 \%)$ \\
DGE & $3(5.5 \%)$ \\
Grade B & $2(3.6 \%)$ \\
Grade C & $1(1.8 \%)$ \\
Intra-abdominal infection & $5(9.1 \%)$ \\
Intra-abdominal haemorrhage & $3(5.5 \%)$ \\
Re-laparotomy & $2(3.6 \%)$ \\
LOS (days), median (IQR) & $14(11-19)$ \\
\hline
\end{tabular}

RPD, robotic pancreaticoduodenectomy; CR-POPF, clinically relevant postoperative pancreatic fistula; DGE, delayed gastric emptying; LOS, length of hospital stay; IQR, inter-quartile.

vascular resections (18). The results showed that RPD with vascular resection had comparable morbidity and mortality rates to RPD, but the combination with vascular resection required a learning curve of 35 cases. Allan et al. reported 70 cases of RPD with concomitant vascular resection with acceptable clinical outcomes (19). However, tumours with obvious vascular involvement were not included in the present study. The main reason was that this study represented the initial experience of our institution during the learning curve.

Several studies have reported the learning curve of RPD (20). Zhang et al. reviewed the first $100 \mathrm{RPD}$ procedures performed by a single surgeon and demonstrated that the learning curve for RPD was 40 cases, with a significant reduction in operative time, EBL and postoperative complications observed afterwards (21). Shi et al. retrospectively reviewed 450 patients who underwent RPD and indicated that 100 cases were required to overcome the learning curve with satisfactory outcomes. The incidence of POPF remarkably decreased after the first 100 cases of RPD, from $30.0 \%$ to $15.1 \%$, and the operative and oncologic outcomes significantly improved after 250 cases of RPD (22). However, the learning curve of RPD is significantly shorter for surgeons with previous LPD experience than for those without previous experience (23) because the operative steps of RPD are similar to those of LPD. Our centre had a step-by-step development for RPD, from OPD, to LPD, RA-LPD and finally total RPD. RA-LPD is a critical transitory stage from LPD to RPD. In addition, familiarity with the established standard process of LPD was of great help in shortening the learning curve of RPD. Over 50 cases of LPD were performed before the first case of RPD in our centre. After the completion of 17 cases of RA-LPD, the operative time remarkably decreased in the total RPD group. The total RPD group seemed to have less EBL than the RA-LPD group, although no significant difference existed. This might be attributed to the superiority of the robotic system in performing gentle and stable dissection of the major vessels and placing precise sutures to control unexpected bleeding. However, more cases are needed to define the learning curve of RPD in this study.

Trocar positions of RPD vary depending on the surgeon's experience in different pancreatic centres. Many studies have reported RPD with 6 to 7 ports, including two assistant ports $(24,25)$. In contrast to those in other studies, the trocar positions of RPD in our centre were similar to those of LPD. In contrast to the two required assistant ports in other studies, only one $12-\mathrm{mm}$ assistant trocar was needed in our study. Giulianotti et al. exchanged R1 and the assistant port during the uncinate process dissection to get closer to the midline and obtain a better operative degree with the harmonic scalpel (2). However, the exchange of trocars was not needed in our operation, which reduced the operative time. The assistant trocar at the intersection of the right midclavicular line and horizontal line at the umbilicus level is convenient for vascular clipping during the dissection of the uncinate process along the SMA to a coincident degree. This approach prevented the use of robotic specialized vascular clips and led to decreased costs. Moreover, it provided a suitable degree to perform side-toside gastrojejunostomy using a $60 \mathrm{~mm}$ linear cutter stapler.

Uncinate process dissection and pancreaticojejunostomy are the most technically challenging steps in RPD. A novel approach for uncinate process dissection and a modified pancreaticojejunostomy were performed in this study. For uncinate process dissection, the right-posterior "SMA-first" approach was performed to determine tumour resectability, separate the uncinate process from the SMA, and ligate the IPDA. After division of the pancreatic neck, the pancreatic head and duodenum were retracted laterally with a $45-60^{\circ}$ anticlockwise rotation, and the SMV was retracted medially. The SMA was then retracted to the right and posterior aspect of the SMV by manipulation, which provided a simultaneously clear view of the SMA and the SMV during 
the separation of the uncinate process. This novel approach was beneficial for radical en bloc resection with an increased $\mathrm{R} 0$ resection rate. For pancreaticojejunostomy, a continuous suture was used for the outside layer of the anastomosis. For the inside layer of the duct-to-mucosa anastomosis, the stitch length was extended from the pancreatic margin to the pancreatic duct, which was different from traditional techniques. The modified technique prevented tearing of the pancreatic parenchyma and pancreatic duct during suturing. It also tightly approximated the jejunum to the pancreatic stump with partial wrapping to avoid a potential space for pancreatic juice to collect postoperatively. A propensity-matched single-centre study by Baimas-George indicated that the POPF incidence of RPD was $10.5 \%$, and RPD offers a decreased incidence of DGE (3\%) and improved oncologic outcomes compared to OPD (26). The overall incidence of POPF (grade B + grade C) and DGE in our centre was $12.7 \%$ and $5.5 \%$, respectively, which were in accordance with the previous study, indicating the safety and feasibility of the technique.

There are several limitations in the present study. This was a retrospective study in a single intuition with a single surgical team and a small sample size. Only patients with periampullary tumours and tumours in the pancreatic head without obvious invasion into the adjacent organs or major vessel involvement were enrolled, which might result in selection bias. A larger sample size or a prospective multicentre randomized controlled trial are required to further validate the efficiency of RPD in the future.

\section{Conclusions}

RPD is a technically feasible procedure for selected patients with periampullary tumours and tumours in the pancreatic head in experienced hands.

\section{Acknowledgments}

Funding: The study was supported by Medical Minimally Invasive Center Program of Fujian Province, China (No. 2017-171), Natural Science Foundation of Fujian Province, China (No. 2018J05131 \& 2020J011013), Key Clinical Specialty Discipline Construction Program of Fujian Province, China (No. 2012-649).

\section{Footnote}

Reporting Checklist: The authors have completed the
STROBE reporting checklist. Available at http://dx.doi. org/10.21037/gs-20-552

Data Sharing Statement: Available at http://dx.doi. org/10.21037/gs-20-552

Conflicts of Interest: All authors have completed the ICMJE uniform disclosure form (available at http://dx.doi. org/10.21037/gs-20-552). The authors have no conflicts of interest to declare.

Ethical Statement: The authors are accountable for all aspects of the work in ensuring that questions related to the accuracy or integrity of any part of the work are appropriately investigated and resolved. The study was conducted in accordance with the Declaration of Helsinki (as revised in 2013). Written informed consent was obtained from the patients for publication of this manuscript and any accompanying images. The study protocol was approved by the ethics committee of Fujian Medical University Union Hospital (2020KY0126).

Open Access Statement: This is an Open Access article distributed in accordance with the Creative Commons Attribution-NonCommercial-NoDerivs 4.0 International License (CC BY-NC-ND 4.0), which permits the noncommercial replication and distribution of the article with the strict proviso that no changes or edits are made and the original work is properly cited (including links to both the formal publication through the relevant DOI and the license). See: https://creativecommons.org/licenses/by-nc-nd/4.0/.

\section{References}

1. Gagner M, Pomp A. Laparoscopic pylorus-preserving pancreatoduodenectomy. Surg Endosc 1994;8:408-10.

2. Giulianotti PC, Mangano A, Bustos RE, et al. Operative technique in robotic pancreaticoduodenectomy (RPD) at University of Illinois at Chicago (UIC): 17 steps standardized technique: Lessons learned since the first worldwide RPD performed in the year 2001. Surg Endosc 2018;32:4329-36.

3. Wang M, Peng B, Liu J, et al. Practice Patterns and Perioperative Outcomes of Laparoscopic Pancreaticoduodenectomy in China: A Retrospective Multicenter Analysis of 1029 Patients. Ann Surg 2021;273:145-53.

4. Rosemurgy A, Ross S, Bourdeau T, et al. Robotic 
Pancreaticoduodenectomy Is the Future: Here and Now. J Am Coll Surg 2019;228:613-24.

5. Nassour I, Wang SC, Christie A, et al. Minimally Invasive Versus Open Pancreaticoduodenectomy: A Propensitymatched Study From a National Cohort of Patients. Ann Surg 2018;268:151-7.

6. Yin Z, Jian Z, Hou B, et al. Surgical and Oncological Outcomes of Laparoscopic Versus Open

Pancreaticoduodenectomy in Patients With Pancreatic Duct Adenocarcinoma. Pancreas 2019;48:861-7.

7. Podda M, Gerardi C, Di Saverio S, et al. Robotic-assisted versus open pancreaticoduodenectomy for patients with benign and malignant periampullary disease: a systematic review and meta-analysis of short-term outcomes. Surg Endosc 2020;34:2390-409.

8. Yan Q, Xu LB, Ren ZF, et al. Robotic versus open pancreaticoduodenectomy: a meta-analysis of short-term outcomes. Surg Endosc 2020;34:501-9.

9. Bassi C, Marchegiani G, Dervenis C, et al. The 2016 update of the International Study Group (ISGPS) definition and grading of postoperative pancreatic fistula: 11 Years After. Surgery 2017;161:584-91.

10. Besselink MG, van Rijssen LB, Bassi C, et al. Definition and classification of chyle leak after pancreatic operation: A consensus statement by the International Study Group on Pancreatic Surgery. Surgery 2017;161:365-72.

11. Wente MN, Bassi C, Dervenis C, et al. Delayed gastric emptying (DGE) after pancreatic surgery: a suggested definition by the International Study Group of Pancreatic Surgery (ISGPS). Surgery 2007;142:761-8.

12. Wente MN, Veit JA, Bassi C, et al. Postpancreatectomy hemorrhage (PPH): an International Study Group of Pancreatic Surgery (ISGPS) definition. Surgery 2007;142:20-5.

13. Shubert CR, Wagie AE, Farnell MB, et al. Clinical Risk Score to Predict Pancreatic Fistula after Pancreatoduodenectomy: Independent External Validation for Open and Laparoscopic Approaches. J Am Coll Surg 2015;221:689-98.

14. Wang SE, Shyr BU, Chen SC, et al. Comparison between robotic and open pancreaticoduodenectomy with modified Blumgart pancreaticojejunostomy: A propensity scorematched study. Surgery 2018;164:1162-7.

15. Kamarajah SK, Bundred J, Marc OS, et al. Robotic versus conventional laparoscopic pancreaticoduodenectomy a systematic review and meta-analysis. Eur J Surg Oncol 2020;46:6-14.

16. Kowalsky SJ, Zenati MS, Steve J, et al. A Combination of Robotic Approach and ERAS Pathway Optimizes
Outcomes and Cost for Pancreatoduodenectomy. Ann Surg 2019;269:1138-45.

17. Shyr BU, Chen SC, Shyr YM, et al. Surgical, survival, and oncological outcomes after vascular resection in robotic and open pancreaticoduodenectomy. Surg Endosc 2020;34:377-83.

18. Beane JD, Zenati M, Hamad A, et al. Robotic pancreatoduodenectomy with vascular resection: Outcomes and learning curve. Surgery 2019;166:8-14.

19. Allan BJ, Novak SM, Hogg ME, et al. Robotic vascular resections during Whipple procedure. J Vis Surg 2018;4:13.

20. Napoli N, Kauffmann EF, Palmeri M, et al. The Learning Curve in Robotic Pancreaticoduodenectomy. Dig Surg 2016;33:299-307.

21. Zhang T, Zhao ZM, Gao YX, et al. The learning curve for a surgeon in robot-assisted laparoscopic pancreaticoduodenectomy: a retrospective study in a highvolume pancreatic center. Surg Endosc 2019;33:2927-33.

22. Shi Y, Wang W, Qiu W, et al. Learning Curve From 450 Cases of Robot-Assisted Pancreaticoduocectomy in a High-Volume Pancreatic Center: Optimization of Operative Procedure and a Retrospective Study. Ann Surg 2019. [Epub ahead of print]. doi: 10.1097/ SLA.0000000000003664.

23. Gall TM, Pencavel TD, Cunningham D, et al. Transition from open and laparoscopic to robotic pancreaticoduodenectomy in a UK tertiary referral hepatobiliary and pancreatic centre - Early experience of robotic pancreaticoduodenectomy. HPB (Oxford) 2020;22:1637-44.

24. Guerra F, Checcacci P, Vegni A, et al. Surgical and oncological outcomes of our first 59 cases of robotic pancreaticoduodenectomy. J Visc Surg 2019;156:185-90.

25. Kim AC, Rist RC, Zureikat AH. Technical Detail for Robot Assisted Pancreaticoduodenectomy. J Vis Exp 2019;(151). doi: 10.3791/60261.

26. Baimas-George M, Watson M, Murphy KJ, et al. Robotic pancreaticoduodenectomy may offer improved oncologic outcomes over open surgery: a propensity-matched singleinstitution study. Surg Endosc 2020;34:3644-9.

Cite this article as: Lin $\mathrm{R}$, Lin $\mathrm{X}$, Pan $\mathrm{M}$, Lu F, Yang $\mathrm{Y}$, Wang C, Fang H, Chen Y, Huang H. Perioperative outcomes of robotic pancreaticoduodenectomy: a single surgeon's experience with 55 consecutive cases. Gland Surg 2021;10(1):122129. doi: $10.21037 / \mathrm{gs}-20-552$ 